\title{
Robotic surgery: applications and cost effectiveness
}

\section{Laura Sigismund Leddy Thomas S Lendvay Richard M Satava}

Department of Urology, University of Washington, Seattle, WA, USA
Correspondence: Laura Sigismund Leddy Department of Urology, University of Washington, Warren G Magnuson Health Sciences Center BB-Wing, Floor II, Box 3565 I0, 1959 NE Pacific Street, Seattle, WA 98195-65I0, USA

Email leddy।@u.washington.edu
This article was published in the following Dove Press journal:

Open Access Surgery

2 September 2010

Number of times this article has been viewed

\begin{abstract}
Robotic surgery has been embraced by many surgical specialties and is being incorporated into an ever growing number of surgical procedures within those specialties. The outcomes and cost data that are available varies greatly between fields. This review examines the history of robotic surgery, the advantages and disadvantages of this technology followed by the applications of robotic surgery in the fields of: urology, gynecology, pediatric surgery and general surgery. Finally, the cost data from the fields of urology and pediatric surgery are reviewed and used to create a model to evaluate the financial impact of a surgical robot on a hospital.
\end{abstract}

Keywords: robotic surgery, cost effectiveness

\section{History}

The impetus to develop robotic surgery platforms evolved from the need to improve the precision of surgical techniques. Over the past 25 years robotic platforms have been introduced to enhance specific surgical procedures. The first surgical robot, the PUMA 560, debuted for the field of neurosurgery. ${ }^{1}$ It was developed for neurosurgical stereotactic maneuvers under computed tomography guidance. Soon thereafter, the PROBOT was developed for the field of urology to guide transurethral resection of the prostate through three-dimensional images created preoperatively ${ }^{2}$ and the ROBODOC was used in Orthopedics to aid in total hip replacement. ${ }^{3}$

The sophistication of robotic technology increased significantly with the concept of robotic telepresence technology, born from a collaboration of the Stanford Research Institute, the Department of Defense and the National Aeronautics and Space Administration. ${ }^{4}$ The original plan for this technology was to provide operative care in the battlefield with surgical robots mounted on armored vehicles while surgeons controlled the robotic arms from distant and protected locations. ${ }^{5}$ A primitive version of this technology, the Aesop (Computer Motion Inc., Goleta, CA) was used to direct the camera during conventional laparoscopic procedures. Followed by the Zeus (Computer Motion Inc., Goleta, CA) a robotic system comprised of three remotely controlled robotic arms that were attached to the surgical table and controlled by the surgeon at a remote console. This brought surgical robots from an entirely passive role in the operating room to a part of the operative environment that was actively controlled by the surgeon. ${ }^{6}$

While research and development in the area of battlefield and remote surgical robots is still ongoing, the major strides in integrating robotic surgery into practice 
Table I Cost of robotic surgery relative to alternatives

\begin{tabular}{|c|c|c|c|c|c|}
\hline & \multicolumn{3}{|c|}{ Total cost } & \multicolumn{2}{|c|}{ Cost as a percent of robotic } \\
\hline & Robotic & Laparoscopic & Open & Laparoscopic & Open \\
\hline \multicolumn{6}{|l|}{ Prostatectomy (Bolenz et $\mathrm{al}^{48}$ ) } \\
\hline Operating room cost & $\$ 2,798$ & $\$ 2,453$ & $\$ 1,611$ & $87.7 \%$ & $57.6 \%$ \\
\hline Hospital cost & 495 & 990 & 990 & $200.0 \%$ & $200.0 \%$ \\
\hline Supplies & 2,015 & 785 & 185 & $39.0 \%$ & $9.2 \%$ \\
\hline Other & $\mathrm{I}, 444$ & $\mathrm{I}, 459$ & $|, 65|$ & $101.0 \%$ & $114.3 \%$ \\
\hline Total (excluding robot) & $\$ 6,752$ & $\$ 5,687$ & $\$ 4,437$ & $84.2 \%$ & $65.7 \%$ \\
\hline Robot amortization per procedure & $\$ 2,698$ & & & & \\
\hline Number of annual procedures & 126 & & & & \\
\hline \multicolumn{6}{|l|}{ Cystectomy (Smith et $\mathrm{al}^{49}$ ) } \\
\hline Operating room cost & $\$ 4,032$ & - & $\$ 2,398$ & - & $59.5 \%$ \\
\hline Hospital cost & $14,4 \mid 8$ & - & 4,982 & - & $|1| 2.8 \%$ \\
\hline Supplies & 7,798 & - & 7,228 & - & $92.7 \%$ \\
\hline Total (excluding robot) & $\$ 16,248$ & - & $\$ 14,608$ & - & $89.9 \%$ \\
\hline \multicolumn{6}{|l|}{ Fundoplication (Anderberg et $\mathrm{al}^{50}$ ) } \\
\hline Operating room cost (anesthesia) & $\$ 3,488$ & $\$ 3,488$ & $\$ 2,174$ & $100.0 \%$ & $62.3 \%$ \\
\hline Hospital cost & 4,015 & 5,494 & 8,347 & $136.8 \%$ & $207.9 \%$ \\
\hline Supplies (instruments) & 2,081 & - & - & $0.0 \%$ & $0.0 \%$ \\
\hline Total (excluding robot) & $\$ 9,584$ & $\$ 8,982$ & $\$ 10,521$ & $93.7 \%$ & $109.8 \%$ \\
\hline Robot amortization per procedure & $\$ 959$ & & & & \\
\hline Number of annual procedures & 330 & & & & \\
\hline
\end{tabular}

Notes: A comparison of the costs related to robotic, laparoscopic and open prostatectomy, cystectomy and fudoplication. In the final two columns the cost breakdown for laparoscopic and open surgery are listed as a percent of the equivalent robotic procedure. In each case it is clear that the cost benefit for robotic surgery occurs within the "Hospital Costs" which is a direct reflection of the length of hospital stay.

are in civilian operating rooms. The first surgical robot using telepresence technology was created for cardiac surgery. ${ }^{6}$ However, telepresence technology is now most pervasive in the fields of gynecology and urology, with limited but increasing applications in general surgery, otolaryngology, cardiac and thoracic surgery. The only US Food and Drug Administration (FDA) approved surgical robot currently on the market is the da Vinci ${ }^{\circledR}$ from Intuitive Surgical Inc. (Sunnyvale, CA).

The focus of the first part of this analysis is on the applications for robotic surgery within the civilian realm and the reasons for its rapid spread. The second part focuses on studies comparing the cost of robotic surgery with conventional laparoscopic surgery and open surgery; along with a discussion of these cost differences and their potential impact on patients and hospitals.

\section{Advantages and disadvantages}

While there is much debate over the outcomes of robotic surgery, there are some benefits to robotic surgery for which the debate is settled. Similar to conventional laparoscopy, robotic-assisted laparoscopic surgery is minimally invasive. Robotic-assisted surgery eliminates the need for large morbid and unsightly incisions and often decreases blood loss, post-operative pain, narcotic use and length of hospital stay in studies across the surgical fields where robotic surgery is currently being applied.

Robotic surgery has several advantages over traditional laparoscopic surgery. These include three-dimensional visualization, mitigation of surgeon tremor, ergonomic and intuitive hand movements, a magnified view and a range of motion approximating the human wrist. The wristed robotic instruments, along with the articulations of the robotic arms, allow the surgeon seven degrees of freedom: the external robotic arms provide three degrees of freedom (insertion, pitch, and yaw) and the Endowrist ${ }^{\circledR}$ mechanism provides four additional degrees of freedom (pitch, yaw rotation and grip). ${ }^{7}$ In contrast, conventional laparoscopic instruments only offer five degrees of freedom: insertion, rotation, pitch, yaw, and grip. The robotic instruments also move as the surgeon's hands would move in an open case, eliminating the counter intuitive fulcrum effect observed in laparoscopic surgery where the surgeon must move his or her hands in the opposite direction of the intended target. Additionally there is scaling down of the surgeons hand movements with the robot to accommodate delicate procedures. ${ }^{7}$ These advantages coupled with a relative ease, and significant improvement in performance and safety of intracorporeal suturing ${ }^{8}$ make the surgical robot a powerful tool in situations requiring a high degree of precision in a tight space. 
Another benefit of robotic-assisted laparoscopic surgery over conventional laparoscopy is its shorter learning curve. Despite the proven benefits for patients, conventional laparoscopy has not been adopted for many applications due to the steep learning curve for surgeons. ${ }^{9}$ The long learning curve is in large part due to the requirement that the surgeon work with long instruments through a fixed entry point on the surface of the body while watching a screen with reduced tactile feed back, leading to diminished fine motor control, tremor amplification and difficult hand-eye coordination. ${ }^{7}$ This is well demonstrated in the fields of urology and gynecology where the laparoscopic prostatectomy and laparoscopic hysterectomy were described in the 1990s. However, the percentage of minimally invasive prostatectomy's and hysterectomies was insignificant until the advent of the surgical robot. This delay in the adoption of minimally invasive techniques in these two very common pelvic surgeries is directly related to their degree of difficulty when performed with conventional laparoscopy, due in large part to the amount of intracorporeal suturing required.

Based on these advantages it would appear that robotic technology should be broadly adopted. However, there are significant obstacles to universal adoption, such as the high cost of the robotic platform, disposable instruments and annual service contracts. Other limitations to robotic-assisted laparoscopic surgery include the lack of haptics, or tactile feedback, ${ }^{9}$ the large and cumbersome footprint of the robot, the fixed positioning of the operating table after the robot has been docked, the longer operative time compared to open surgery, and the limited outcomes data.

The following sections will delve into the many applications of robotic surgery across multiple surgical disciplines, and further weigh the pros and cons of this new technology.

\section{Applications and outcomes Applications and outcomes in urology}

Robotic technology has penetrated the deepest in the field of urology, driven by the amount of suturing required in many urologic procedures combined with urologist's comfort with minimally invasive procedures. This comfort, developed first in the realm of endourology for stone and cancer therapy, has been a significant driving force in the wide and rapid adoption of robotic surgery in the field of urology. ${ }^{8}$

The most common application for robotic surgery in the field of urology is the radical prostatectomy. The use of the robot for radical prostatectomy increased from $1 \%$ of all prostatectomies performed in the United States in 2001 to almost $40 \%$ in $2006-2007 .{ }^{10}$ More than $50 \%$ of all prostatectomies performed in the US in 2009 will be robot assisted. ${ }^{11}$

Data comparing the outcomes of robotic assisted prostatectomy to open prostatectomy are being generated and debated within the urology community. Hu et al at the Division of Urology at Brigham and Women's Hospital in Boston, Massachusetts identified 8837 men who underwent radical prostatectomy for prostate cancer, from 2003 to 2007 in order to perform a retrospective review and identify potential differences in outcomes between minimally invasive radical prostatectomy with and without robotic assistance and the gold standard, open prostatectomy. They found that men who underwent minimally invasive radical prostatectomy experienced a shorter length of stay, lower rates of blood transfusion, fewer respiratory and miscellaneous surgical complications and fewer strictures. Both groups had similar postoperative use of additional cancer therapies suggesting that minimally invasive radical prostatectomy is equivalent to the gold standard as a cancer therapy. The minimally invasive group had a $2.6 \%$ higher risk of genitourinary complications, a $3.7 \%$ higher risk of incontinence and a $7.6 \%$ higher risk of erectile dysfunction. ${ }^{10}$

Ficarra, et al have effectively synthesized the available outcomes data pertaining to minimally invasive radical prostatectomy and published their findings in European Urology. ${ }^{12}$ The review includes 37 studies comparing open, laparoscopic and robot-assisted radical prostatectomy. The study found again that both laparoscopic prostatectomy and robotic assisted laparoscopic prostatectomy had significantly lower blood loss and rates of transfusion, shorter hospital stay and lower complication rates. They found that an open prostatectomy required less operative time than either conventional laparoscopic and robotic prostatectomy. The continence and potency rates appeared similar between all three surgical modalities. The oncologic outcome most frequently evaluated, the rate of positive surgical margins, again was similar between open, conventional laparoscopy and robotic surgery. ${ }^{12}$

It therefore appears that robotic prostatectomy is a reasonable choice for prostate cancer therapy and a therapy that is worthy of comparison against the current standard of care in operative therapy for prostate cancer: the radical retropubic prostatectomy. The urologic community is awaiting five- and ten-year follow up data for PSA recurrence as a marker of cancer control for this slow growing cancer. 
In a recent comparison of 104 open radical cystectomies versus 83 robotic cystectomies the operative time, lymph node yield and rate of positive margins were similar while the robotic procedure was favorable in terms of less blood loss, shorter length of stay and lower major complication rates. ${ }^{13}$ Pruthi et al at the University of North Carolina at Chapel Hill have recently published their series of 100 consecutive patients undergoing robotic radical cystectomy with a $0 \%$ positive margin rate and a $20 \%$ positive lymph node rate, 41 postoperative complications were identified in 36 of 100 patients. They reported an $8 \%$ rate of major complications and $11 \%$ rate of readmission. The mean follow up was 21 months at which time 15 patients had disease recurrence and six patients had died. While there was not a comparison group of open radical cystectomy patients it was concluded that robotic radical cystectomy could be performed without compromising surgical outcomes or pathological results. ${ }^{14}$ This study and others like it do not assess long-term cancer-related outcomes. The same group had previously published data on the learning curve for robotic cystectomy. They found that after 20 cases the operative time and blood loss decrease to a plateau. ${ }^{15}$

Kural et al compared a series of 20 laparoscopic partial nephrectomies with 11 robotic-assisted partial nephrectomies finding a trend toward a shorter operative time and less blood loss in the robotic cases though these were not significant, however they did find that the warm ischemia time was significantly shorter for the robotic cases. There was one focal positive margin the conventional laparoscopic group and no positive surgical margins in the robotic group. ${ }^{16}$

In addition to using the da Vinci surgical system as a surgical therapy for radical prostatectomy, cystectomy and partial nephrectomy urologists are using the robot for in the fields of male infertility, female incontinence and in pediatric urology for pyeloplasty and ureteral reimplantation.

The published data on these procedures is less extensive, but initial outcomes appear comparable or even favorable to open procedures.

\section{Applications and outcomes in gynecology}

Robotic surgery has also found a foothold within the field of gynecology, where minimally invasive procedures such as the laparoscopic hysterectomy have shown a faster recovery, shorter hospitalization, improved cosmesis, decreased blood loss and less postoperative pain. ${ }^{17,18}$ Despite the proven benefits of minimally invasive surgery, the overwhelming majority of these procedures and others involving extensive suturing (eg, myomectomy and sacrocolpopexy) are still performed with a laparotomy. ${ }^{9}$ A large population based study of over 500,000 women undergoing hysterectomy in 2003 demonstrated that only $11.8 \%$ were performed laparoscopically, despite proven benefits supporting this technique. ${ }^{19}$ This is again due to the steep learning curve for conventional laparoscopic surgery ${ }^{9}$ particularly for procedures requiring significant intracorporeal suturing. The da Vinci surgical system is being heralded as a means of overcoming these obstacles while extending the benefits of minimally invasive surgery to more patients. ${ }^{6,9}$

In 2005, five years after its initial approval in the field of urology, the FDA approved the use of the da Vinci surgical system in gynecologic surgery. ${ }^{6}$ Since then, the surgical robot has been used in a number of gynecologic procedures including; hysterectomy (with and without bilateral salpingooophorectomy), myomectomy, tubal reanastomoses, pelvic and paraaortic lymph node dissection, and sacrocolpopexy. ${ }^{6}$

There are a number of small case series of robotic hysterectomy reported in the gynecologic literature dating back to 2002, each one involving less than 20 patients. The largest published series is from the Mayo Clinic, Scottsdale involving a retrospective review of 91 patients who underwent robotic hysterectomy without lymphadenectomy for both benign and malignant pathology. There were no conversions to open surgery and no bladder or ureteral injuries. There was not an open or conventional laparoscopic comparison group in this study. ${ }^{20}$ Payne and Dauterive performed a retrospective review of 100 hysterectomies performed before and after implementation of the robotic program in their community practice. The study found a significantly lower rate of conversion to open and a lower mean blood loss in the robotic group. The incidence of adverse events was similar between the two groups. ${ }^{21}$

Several small case series have also been published on the use of robotic surgery as treatment and staging for gynecologic malignancies such as radical hysterectomy for endometrial and cervical cancers. Again these studies involve less than 20 patients but seem to show that the robotic cases require a longer operative time than open cases but yield similar or higher numbers of lymph nodes..$^{22,23,24}$ Currently no five-year survival data exists to assess the efficacy of the robotic platform as a means of cancer control.

\section{Applications in pediatric surgery}

The da Vinci robot has also been approved by the FDA for use in the field of pediatric surgery. Case series and/or small 
studies comparing robotic surgery to open or conventional laparoscopic surgery exist for pediatric populations in the fields of general surgery for Nissen fundoplication, urology for dismembered pyeloplasty, nephrectomy and calyceal diverticulectomy and in cardiothoracic surgery for PDA (patent ductus arteriosis) closure. Additionally, a number of complex procedures have been described in case reports or small case series within the surgical literature. ${ }^{25}$ These include bladder augmentation, Mitrofanoff appendicovesicostomy, partial nephrectomy, ureteral reimplantation, atrial septal defect closure, choledochal cyst excision, Kasai portoenterostomy, Bochdalek congenital diaphragmatic hernia repair, seminal vesicle cyst removal, Mullerian and Wolffian duct remnant removal and reoperative pyeloplasty. ${ }^{25}$

Published post operative data indicates a trend toward less post operative pain and a shorter length of hospital stay for robotic pyeloplasty and Nissen fundoplication. ${ }^{26,27,28}$ While the reported complication rates for robotic assisted pediatric surgery appear to be acceptably low. The rate of intraoperative conversion to open surgery ranged from $0 \%-13 \%$. This wide range reflects varying levels of surgical skills as well as varying complexity of the procedure being performed. ${ }^{25}$ Quoted complication rates for robotic surgery were of a similar type and rate to corresponding conventional laparoscopic procedures, thus reported complications were not deemed to be the direct cause of the use of the robot. ${ }^{25}$

Current data demonstrates that while robotic pyeloplasties are $20 \%$ longer than open pyeloplasties there is no difference in the total operating room time for robotically assisted pyeloplasty and conventional laparoscopic pyeloplasty. ${ }^{27}$ This did not hold true for robotically assisted patent ductus arteriosus closure when compared with traditional video-thoracoscopic surgery due to the complexity of robot placement. ${ }^{29}$

Small case series demonstrate efficacy for several pediatric robotic procedures, including 24-hour $\mathrm{pH}$ measurements after fundoplication $^{30}$ and renal ultrasound examination or renal clearance testing after pyeloplasty. ${ }^{26,27}$ However, long-term outcomes data is not yet available.

While published data are still slim on the outcomes of robotic surgery in the pediatric population, there are trends toward the same benefits that make the surgical robot useful within adult populations. These data need to be considered in the setting of small case series where a relatively new procedure is being compared to a procedure at which the surgeon is well practiced.

\section{Applications in general surgery}

The field of general surgery has been much slower to adopt robot-assisted laparoscopic surgery. Published data in this field is limited mostly to case series and discussions of surgical techniques on an increasing array of procedures. The areas of greatest interest for integrating robotic technology into the field of general surgery lie in foregut surgery, hepatobiliary surgery, endocrine surgery, colorectal surgery and bariatric surgery. ${ }^{16}$

In foregut surgery there are small case series comparing robotic and conventional laparoscopic Nissen fundoplication, robotic and conventional laparoscopic Heller myotomy with comparable results, ${ }^{31-34}$ as well as initial series of robotic gasterectomy and subtotal gasterectomy for gastric cancer. ${ }^{35}$ Long-term cancer-free survival data is not yet available. Case reports are available indicating the safety and feasibility of the technically complex esophagectomy. ${ }^{36}$ Comparison studies with the open esophagectomy have the potential to show a benefit due to the high morbidity of the open procedure.

In hepatobiliary surgery, studies comparing the commonly performed and relatively straightforward laparoscopic cholecystectomy with robotic cholecystectomy do not demonstrate any benefit to the robotic procedure, though they do confirm its safety and efficacy. ${ }^{37,38}$ Robotic hepatectomies, hepatic lobectomies and segmentectomies have all been described and appear safe and feasible. ${ }^{39}$ Due to their complexity they are rarely performed with conventional laparoscopy.

In initial studies robotic technology appears less useful in endocrine surgery. For instance a small randomized control trial comparing laparoscopic to robotic adrenalectomy found the robotic approach had more morbidity, cost and operative time. ${ }^{40}$ Case series have been published on robotic pancreatectomy, pancreaticoduodenectomy and transaxillary thyroidectomy though none involved a comparison to open surgery. ${ }^{39,41-43}$

In colorectal surgery a small comparative trial of robotic versus laparoscopic total mesorectal excision for rectal cancer demonstrated a possible benefit of robotic surgery with a shorter length of hospital stay and similarly low complication rate between the two arms. ${ }^{44}$ However robotic total colectomy is likely to be more difficult than conventional laparoscopic colectomy due to the need to access multiple quadrants of the abdomen during this procedure requiring redocking the robot mid-case. ${ }^{16}$

There are early indications that robotic surgery will offer some benefits in the more complex bariatric procedures that require more complex intracorporeal sewing..$^{45,46}$ No benefit 
is seen in the simpler procedures that do not require suturing such as placement of the adjustable gastric band. ${ }^{47}$

The general surgeons' hesitancy in embracing robotic technology may be a combination of the large territory covered in many abdominal surgeries coupled with the current lack of a robotic device for performing stapled bowel anastomoses, ${ }^{16}$ yet such a technology promises to be forthcoming. While the adoption of robotic technology has been relatively slow in the field of general surgery, general surgeons continue to seek useful applications for the technology.

\section{Review of cost data}

It seems intuitive that robotic surgery would be more costly than open surgery as it requires a large initial investment on the order of US\$1-2 million and ongoing annual maintenance costs on the order of US $\$ 340,000^{48}$ that are not present in open surgery. In addition, robotic surgery requires disposable or limited use instruments (eg, shears, needle drivers, graspers, forceps) at a cost of approximately US $\$ 2,200$ per instrument every 10 surgeries ${ }^{48}$ versus the mostly reusable instruments in open surgery. Robotic surgery takes on average as long or longer than the corresponding open procedure, thus there is not a cost savings in operating room or anesthesia time. This leaves the only potential for cost savings in a decreased length of hospital stay.

Several cost comparison studies exist that demonstrate the relative cost drivers of robotic surgery versus open surgery. Three of these studies are detailed below:

The largest cost comparison study was recently published in European Urology by Bolenz, et al from the University of Texas Southwestern and Mannheim Medical Center at the University of Heidelberg. The study compared operating costs (not including maintenance and equipment purchase) of robotic (RALP), laparoscopic (LRP), and open radical prostatectomy (RRP) for prostate cancer in a sample of 643 consecutive patients treated at Southwestern Medical Center in Dallas, Texas. Results showed that the cost of RALP was $50 \%$ higher than the cost of RRP even before the cost of purchasing and maintaining the robot was factored in to the calculations. The median cost for the RALP was US $\$ 6,752$, followed by LRP at US $\$ 5,687$ and RRP at US $\$ 4,437$ (all adjusted to 2007 dollars). RALP had higher surgical supply costs and higher OR cost due to increased average length of procedure. The one cost benefit for RALP was the shorter average length of hospital stay (one day) relative to LRP and RRP (two days). However, the shorter RALP hospital stay relative to LRP and RRP did not make up for the RALP higher operating costs, even before considering the additional cost for the purchase and maintenance of the robot. The additional cost for the purchase and maintenance of the robot $(\$ 340,000$ per year when amortized over a presumed $7 \mathrm{yr}$ life of the robot) would add an additional $\$ 2698$ per patient undergoing a RALP (assuming 126 cases per year) ${ }^{48}$

A similar cost comparison study was recently completed for robotic versus open radical cystectomy for bladder cancer at the University of North Carolina at Chapel Hill. In this study the 20 most recent cases of robotic cystectomy were compared with the 20 most recent cases of open cystectomy. The total cost (including base OR costs, OR disposable equipment costs, amortized purchase cost of the robot distributed over 5 years and yearly maintenance costs) of the robotic radical cystectomy was $\$ 1,640$ more than the open radical cystectomy $(\$ 16,248$ versus $\$ 14,608)$. In the breakdown of the costs the majority of the difference came in the higher mean fixed OR costs for robotic cases (OR disposable equipment costs, amortized purchase cost and yearly maintenance cost distributed over 288 cases per year). The OR variable costs were also slightly higher for the robotic cystectomy due to the increased length of these cases. Similar to the robotic prostatectomy cost data, there was some cost savings due to a shorter postoperative stay as well as a lower frequency of postoperative transfusion after open cystectomy. While these savings were not enough to overcome the increased OR costs of the robotic cystectomy, the cost differential of $\$ 1,640$ per surgery was much closer than the robotic prostatectomy cost study. ${ }^{49}$

Robotic versus open surgery cost comparison studies also exist in the medical literature outside the field of urology. For instance, a recent prospective cost comparison study performed at University Hospital in Lund Sweden compared the cost of the first 14 pediatric robotic assisted fundoplications with the cost of the 10 most recent fundoplications performed using open and laparoscopic techniques. The study found that the mean cost of the robotic fundoplication ( $€ 9,584$, excluding purchase cost and annual maintenance) was $7 \%$ higher than the mean cost of the equivalent laparoscopic procedure $(€ 8,982)$ and $9 \%$ lower than the mean cost for the open surgical procedure $(€ 10,521)$. The higher intraoperative cost of the robotic procedure was offset by a much lower average length of hospital stay (an average of 3.8 days for robotic surgery versus 5.2 for laparoscopic and 7.9 days for open surgery). In other words, the difference in length of hospital stay was enough to recuperate the increased variable surgical costs for the robotic procedure. If the amortized cost of the initial purchase and annual maintenance costs of the robot are added back in (€959 if 330 robotic cases are performed annually) the cost of a robotic-assisted fundoplica- 
tion is equivalent to that of an open fundoplication ( $€ 10,543$ for robotic versus $€ 10,521$ for open). ${ }^{50}$

In reviewing a small population of robotic prostatectomies compared with open prostatectomies completed at the University of Washington in 2009, the average charge (intraoperative cost and hospital cost) to the patient for a RALP is $20 \%$ higher than for an RRP. This does not include the amortized cost of the robot or a share of the yearly maintenance cost, as this is not charged to the patient.

We are currently in a phase of rapid growth and dissemination of the applications for robotic surgical technology within a growing number of surgical fields. Yet with the rising cost of health care a constant topic of discussion it is important to have a means of evaluating new technologies not only by the outcomes for patients but also on their effect on economics of health care as a whole. The only cost advantage for robotic surgery supported by the medical literature a reduction in the number of days spent in the hospital. This becomes a cost savings for the patient when enough nights in the hospital are saved to overcome the increased cost of the robotic procedure.

A second perspective to take when evaluating the cost of robotic surgery is that of the hospital. When a hospital is deciding whether the purchase of a robot is appropriate for their institution, it is looking at a large upfront investment and yearly maintenance costs, which are not directly passed on to patients when they are charged for robotic procedures. How does a hospital decide whether the purchase of a surgical robot will be a sound financial decision? Again, it is the number of nights in the hospital that is saved that is the determining factor.

If a hospital is constrained in the number of in-patient procedures they can perform due to a shortage of beds, the investment in a surgical robotic could generate significant financial returns due to the reduced hospital stay time required for patients who undergo robotics procedures relative to open procedures. For instance, if we assume a hospital can use the robot on 280 cases annually and that these procedures reduce patient hospital stay time by one day relative to open procedures, the hospital would gain 280 bed days of capacity. The hospital could fill those beds by conducting more procedures. If the average in-patient procedure requires one day of hospital stay, the hospital could perform 280 additional procedures in a year. Finally, if each of these procedures generates $\$ 4,000$ of contribution margin to the hospital, total value created for the hospital would exceed $\$ 1.1$ million dollars annually. Of course, we would need to factor in the up-front cost of acquiring the robot plus the ongoing maintenance costs. If the up-front cost for a robot is $\$ 1.5$ million and ongoing variable costs (eg, maintenance and disposable instruments) are $\$ 0.4$ million annually, the robot would pay for itself in just over two years ( $\$ 1.5 /$ [ $\$ 1.1-\$ 0.4]=\sim 2.1$ years), at a $48 \%$ return on investment (ROI). Two other examples of this same cost analysis are demonstrated in Table 2. Example A demonstrates a scenario in which a hospital would not benefit from the purchase of a surgical robot. Example B demonstrates

Table 2 Robot break-even analysis (years to pay off robot acquisition)

\begin{tabular}{|c|c|c|c|}
\hline & \multicolumn{3}{|c|}{ Break-even calculation (illustrative) } \\
\hline & Example A & Example B & Basis for assumption \\
\hline Robotic cases per year & 126 & 330 & Bolenz et $\mathrm{al}^{48}$; Anderberg et $\mathrm{al}^{50}$ \\
\hline Hospital days saved with robotic procedure (per case) & 1.0 & 4.0 & Bolenz et $\mathrm{al}^{48}$; Anderberg et $\mathrm{al}^{50}$ \\
\hline Hospital days saved (additional over-night capacity available) & 126 & 1,320 & \\
\hline Nights stay after average surgical procedure & 1.0 & 1.0 & Varies by hospital and procedure \\
\hline Number of procedures made possible by freed up beds & 126 & $\mathrm{I}, 320$ & \\
\hline Average contribution margin per procedure (including stay) & $\$ 3,500$ & $\$ 3,500$ & Varies by hospital and procedure \\
\hline Annual value $(\mathrm{CM})$ created from increased bed capacity & $\$ 44 I, 000$ & $\$ 4,620,000$ & \\
\hline \multicolumn{4}{|l|}{ Less annual incremental costs of using robot } \\
\hline Maintenance & $(\$ 340,000)$ & $(\$ 340,000)$ & Bolenz et $\mathrm{al}^{48}$ \\
\hline Disposable/limited use instruments & $(\$ 60,000)$ & $(\$ 60,000)$ & Ficarra et al ${ }^{12}$ \\
\hline Net annual benefit (capacity value minus incremental cost) & $\$ 41,000$ & $\$ 4,220,000$ & \\
\hline Upfront investment to acquire and install robot & $\$ 1,500,000$ & $\$ 1,500,000$ & Bolenz et al ${ }^{48}$ \\
\hline Years to pay off acquisition & 36.6 & 0.4 & \\
\hline $\mathrm{ROI}$ & $2.7 \%$ & $281.3 \%$ & \\
\hline
\end{tabular}

Notes: A model to evaluate the financial impact of a surgical robot on a hospital. A key assumption in this model is that surgical procedures can be gained by increasing hospital bed availability. The numbers in italics represent the variables for the model. The reference supporting each variable is listed in the final column. The "Years to pay off acquisition" decreases and the "Return on Investment (ROI)" increases as the number of robotic cases per year increases, the hospital days saved with robotic procedure increases and the contribution margin per procedure increases. Example A is based on the number of robotic cases per year and number of hospital days saved with robotic prostatectomy in the paper by Bolenz et al. ${ }^{48}$ Example B is based on the number of robotic cases per year and number of hospital days saved with robotic fundoplication in the paper by Anderberg et al. ${ }^{50}$ The average contribution margin to the hospital is an arbitrary value that is fixed as equal in each example as are the maintenance, instrument and initial investment costs. Based on these hypothetical situations the ROI in Example A is very poor at $2.7 \%$ with a prohibitively high number of years to pay of acquisition. Whereas, in Example B the ROI is $281.3 \%$ and the initial investment would be paid off in 0.4 years. 
a scenario where a hospital would benefit financially from the purchase of a robot. In reality, most hospitals perform a variety of surgical procedures robotically and would thus fall in between these two examples.

Another element of the societal cost for open vs robotic surgery involves the convalescence of the patient after hospital discharge. There has been very little studied about the impact on economics that a more rapid return to work provides. In open surgery, the potentially more morbid incisions and postoperative pain, may delay a patient's return to the workforce compared to robotic surgery. The financial impact of a patient who is unable to return to work while recovering from surgery can negatively impact productivity in business. Until this aspect of the financial balance is evaluated thoroughly, the economic burden of robotics cannot be confirmed.

Thoughtful studies are being conducted and need to continue to be produced in order for robotic technology to be evaluated against current standards of care with regard to both short term and long term outcomes. The early outcomes evaluations seem to indicate that robotic surgery is safe and effective as a treatment after physicians perform a predictable numbers of cases. However, in our current economic climate it is equally important for medical institutions and patients alike to consider the financial impact of treatment decisions. Key emerging observations regarding the cost of robotic surgery across all fields include:

1. The fixed (equipment and maintenance) and variable (instruments) costs for robotic surgery are higher than both conventional laparoscopic or open surgery.

2. The OR costs of robotic surgery are higher due to an increased length of the procedure over open surgery (though not over conventional laparoscopic surgery).

3. When the total (fixed, variable, OR, and hospital stay) costs for robotic surgery and open surgery are comparable it is due to a considerable shortening of the length of hospital stay after the robotic surgery resulting in total cost savings.

4. Conventional laparoscopic surgery shares the minimally invasive benefits of robotic surgery and is less expensive due to lower variable costs; however, there remain many procedures that the majority of surgeons are not able to perform laparoscopically due to the prohibitively long learning curve. The learning curve for the procedures studied is shorter with robotic surgery and conventional laparoscopic surgery.

\section{Conclusion}

For procedures where a minimally invasive approach can be shown to significantly decrease hospital stay and for which conventional laparoscopy has a prohibitively long learning curve, robotic surgery may be cost effective. While patients see the additional benefits of robotic surgery as a minimally invasive technique that will become more widely available than conventional lap due to the shortened learning curve, hospitals may see additional financial benefits as the technique becomes more cost effective. Potential future cost savings for both hospitals and patients can be found in shorter operative times as surgeons complete their learning curves. This will also allow more procedures to be performed, which spreads the fixed costs of the robot over more patients. Also improved surgical technique coupled with shorter OR times could lead to even shorter hospital stays decreasing costs to patients and allowing for further revenue opportunities for hospitals. Finally, as robotic technology expands its cost, just like the cost of all other technologies before it, will decrease over time with the inevitable advent of competitors in the market place. It may be this factor in the end that provides the greatest cost savings to both patients and hospitals allowing more patients the indisputable benefits of minimally invasive surgery within an economically responsible framework.

\section{Disclosure}

The authors report no conflicts of interest in this work.

\section{References}

1. Kwoh YS, Hou J, Jonckheere EA, Hayati S. A robot with improved absolute positioning accuracy for CT guided stereotactic brain surgery. IEEE Trans Biomed Eng. 1988;35(2):153-160.

2. Davies BL, Hibberd RD, Coptcoat MJ, Wickham JE. A surgeon robot prostatectomy - a laboratory evaluation. J Med Eng Technol. 1989;13(6):273-277.

3. Bauer A, Borner M, Lahmer A. Clinical experience with a medical robotic system for total hip replacement. In: Nolte LP, Ganz R, editors. Computer Assisted Orthopedic Surgery. Bern, Germany: Hogrefe and Huber; 1999.

4. Satava RM. Robotic surgery: from past to future - a personal journey. Surg Clin North Am. 2003;83(6):1491-1500, xii.

5. Diodato MD Jr, Damiano RJ Jr. Robotic cardiac surgery: overview. Surg Clin North Am. 2003;83(6):1351-1367, ix.

6. Advincula AP, Song A. The role of robotic surgery in gynecology. Curr Opin Obstet Gynecol. 2007;19(4):331-336.

7. Chen CC, Falcone T. Robotic gynecologic surgery: past, present, and future. Clin Obstet Gynecol. 2009;52(3):335-343.

8. Stefanidis D, Wang F, Korndorffer JR Jr, et al. Robotic assistance improves intracorporeal suturing performance and safety in the operating room while decreasing operator workload. Surg Endosc. 2010;24(2):377-382. Epub 2009 Jun 18.

9. Visco AG, Advincula AP. Robotic gynecologic surgery. Obstet Gynecol. 2008;112(6):1369-1384.

10. Hu JC, Gu X, Lipsitz SR, et al. Comparative effectiveness of minimally invasive vs open radical prostatectomy. JAMA. 2009; 302(14):1557-1564.

11. Pruthi RS, Wallen EM. Current status of robotic prostatectomy: promises fulfilled. J Urol. 2009;181(6): 2420-2421. 
12. Ficarra V, Novara G, Artibani W, et al. Retropubic, laparoscopic, and robot-assisted radical prostatectomy: a systematic review and cumulative analysis of comparative studies. Eur Urol. 2009;55(5):1037-1063.

13. Ng CK, Kauffman EC, Lee MM, et al. A comparison of postoperative complications in open versus robotic cystectomy. Eur Urol. 2010;57(2):274-281.

14. Pruthi RS. Robotic radical cystectomy for bladder cancer: Surgical and pathological outcomes in 100 consecutive cases. $J$ Urol. 2010;183:510-515.

15. Pruthi RS, Smith A, Wallen EM. Evaluating the learning curve for robot-assisted laparoscopic radical cystectomy. J Endourol. 2008;22(11):2469-2474.

16. Kural AR, Atug F, Tufek I, et al. Robot-assisted partial nephrectomy versus laparoscopic partial nephrectomy: comparison of outcomes. J Endourol. 2009;23(9):1491-1497.

17. Mais V, Ajossa S, Guerriero S, MAscia M, Solla E, Melis GB. Laparoscopic versus abdominal myomectomy: a prospective, randomized trial to evaluate benefits in early outcome. Am J Obstet Gynecol.1996;174(2):654-658.

18. Paraiso MF, Walters MD, Rackley RR, Melek S, Hugney C. Laparoscopic and abdominal sacral colpopexies: a comparative cohort study. Am J Obstet Gynecol. 2005;192(5):1752-1758.

19. Wu JM, Wechter ME, Geller EJ, et al. Hysterectomy rates in the United States, 2003. Obstet Gynecol. 2007;110(5):1091-1095.

20. Kho RM, Hilger WS, Heutz JG, Magtibay PM, Magrina JF. Robotic hysterectomy: technique and initial outcomes. Am J Obstet Gynecol. 2007;197(1):113 e1-e4.

21. Payne TN, Dauterive FR. A comparison of total laparoscopic hysterectomy to robotically assisted hysterectomy: surgical outcomes in a community practice. J Minim Invasive Gynecol. 2008;15(3):286-291.

22. Magrina JF, Kho RM, Weaver AL, Montero RP, Magtibay PM. Robotic radical hysterectomy: comparison with laparoscopy and laparotomy. Gynecol Oncol. 2008;109(1):86-91.

23. Boggess JF, Gehrig PA, Cantrell L, et al. A case-control study of robotassisted type III radical hysterectomy with pelvic lymph node dissection compared with open radical hysterectomy. Am J Obstet Gynecol. 2008;199(4):357 e1-e7.

24. Boggess JF, Gehrig PA, Cantrell L, et al. A comparative study of 3 surgical methods for hysterectomy with staging for endometrial cancer: robotic assistance, laparoscopy, laparotomy. Am J Obstet Gynecol. 2008;199(4):360 e1-e9.

25. van Haasteren G, Levine S, Hayes W. Pediatric robotic surgery: early assessment. Pediatrics. 2009;124(6):1642-1649.

26. Lee RS, Retik AB, Borer JG, Peters CA. Pediatric robot assisted laparoscopic dismembered pyeloplasty: comparison with a cohort of open surgery. J Urol. 2006;175(2):683-687.

27. Franco I, Dyer LL, Zelkovic P. Laparoscopic pyeloplasty in the pediatric patient: hand sewn anastomosis versus robotic assisted anastomosis - is there a difference? J Urol. 2007;178(4):1483-1486.

28. Yee DS, Shanberg AM, Duel BP, Rodriguez E, Eichel L, Rajpoot D. Initial comparison of robotic-assisted laparoscopic versus open pyeloplasty in children. Urology. 2006;67(3):599-602.

29. Le Bret E, Papadatos S, Folliguet T, et al. Interruption of patent ductus arteriosus in children: robotically assisted versus videothoracoscopic surgery. J Thorac Cardiovasc Surg. 2002;123(5):973-976.

30. Anderberg M, Kockum CC, Arnbjornsson E. Robotic fundoplication in children. Pediatr Surg Int. 2007;23(2):123-127.

Open Access Surgery

\section{Publish your work in this journal}

Open Access Surgery is an international, peer-reviewed, open access journal that focuses on all aspects of surgical procedures and interventions. Patient care around the peri-operative period and patient outcomes post surgery are key topics. All grades of surgery from minor cosmetic interventions to major surgical procedures are covered. Novel techniques Submit your manuscript here: http://www.dovepress.com/open-access-surgery-journal
31. Muller-Stich BP, Reiter MA, Wente MN, et al. Robot-assisted versus conventional laparoscopic fundoplication: short-term outcome of a pilot randomized controlled trial. Surg Endosc. 2007;21(10):1800-1805.

32. Galvani C, Gorodner MV, Moser F, Baptista M, Donahue P, Horgan S. Laparoscopic Heller myotomy for achalasia facilitated by robotic assistance. Surg Endosc. 2006;20(7):1105-1112.

33. Melvin WS, Dundon JM, Talamini M, Horgan S. Computer-enhanced robotic telesurgery minimizes esophageal perforation during Heller myotomy. Surgery. 2005;138(4):553-559.

34. Horgan S, Galvani C, Gorodner MV, et al. Robotic-assisted Heller myotomy versus laparoscopic Heller myotomy for the treatment of esophageal achalasia: multicenter study. J Gastrointest Surg. 2005;9(8):1020-1030.

35. Song J, Kang WH, Oh SJ, Hyung WJ, Choi SH, Noh SH. Role of robotic gastrectomy using da Vinci system compared with laparoscopic gastrectomy: initial experience of 20 consecutive cases. Surg Endosc. 2009;23(6):1204-1211.

36. Galvani CA, Gorodner MV, Moser F. Robotically assisted laparoscopic transhiatal esophagectomy. Surg Endosc. 2008;22(1):188-195.

37. Hazey JW, Melvin WS. Robot-assisted general surgery. Semin Laparosc Surg. 2004;11(2):107-112.

38. Ballantyne GH. Telerobotic gastrointestinal surgery: phase 2 - safety and efficacy. Surg Endosc. 2007;21(7):1054-1062.

39. Giulianotti PC, Kuechle J, Salehi P, et al. Robotic-assisted laparoscopic distal pancreatectomy of a redo case combined with autologous islet transplantation for chronic pancreatitis. Pancreas. 2009;38(1):105-107.

40. Morino M, Benincà G, Giraudo G, Del Genio GM, Rebecchi F, Garrone C. Robot-assisted vs laparoscopic adrenalectomy: a prospective randomized controlled trial. Surg Endosc. 2004;18(12): 1742-1746.

41. Melvin WS, Needleman BJ, Krause KR, et al. Computer-enhanced robotic telesurgery. Initial experience in foregut surgery. Surg Endosc. 2002;16(12):1790-1792.

42. Melvin WS, Needleman BJ, Krause KR, Ellison EC. Robotic resection of pancreatic neuroendocrine tumor. J Laparoendosc Adv Surg Tech A. 2003;13(1):33-36.

43. Chung W. Transaxillary robotic thyroidectomy. Houston, TX: Pan American Robotic Oncology Symposium; 2009.

44. Baik SH, Ko YT, Kang CM, et al. Robotic tumor-specific mesorectal excision of rectal cancer: short-term outcome of a pilot randomized trial. Surg Endosc. 2008;22(7):1601-1608.

45. Yu SC, Clapp BL, Lee MJ, Albrecht WC, Scarborough TK, Wilson EB. Robotic assistance provides excellent outcomes during the learning curve for laparoscopic Roux-en-Y gastric bypass: results from 100 robotic-assisted gastric bypasses. Am J Surg. 2006;192(6): 746-749.

46. Snyder B, Wilson T, Scarborough T, et al. Lowering gastrointestinal leak rates: a comparative analysis of robotic and laparoscopic gastric bypass. Robotic Surgery. 2008;2:159-163.

47. Muhlmann G, Klaus A, Kirchmayr W, et al. Da Vinic robotic-assisted laparoscopic bariatric surgery: Is it justified in a routine setting? Obes Surg. 2003;13(6):848-854.

48. Bolenz C, Gupta A, Hotze T, et al. Cost comparison of robotic, laparoscopic, and open radical prostatectomy for prostate cancer. Eur Urol. 2010;57(3):453-458.

49. Smith A, Kurpad R, Lal A, et al. Cost analysis of robotic versus open radical cystectomy for bladder cancer. J Urol. 2010;183(2):505-509.

50. Anderberg M, Kockum CC, Arnbjornsson E. Paediatric robotic surgery in clinical practice: a cost analysis. Eur J Pediatr Surg. 2009;19(5): 311-315.

\section{Dovepress}

and the utilization of new instruments and materials, including implants and prostheses that optimize outcomes constitute major areas of interest. The manuscript management system is completely online and includes a very quick and fair peer-review system. Visit http://www.dovepress.com/ testimonials.php to read real quotes from published authors. 